Modeling, Identification and Control, Vol. 42, No. 3, 2021, pp. 131-141, ISSN 1890-1328

\title{
Optimal control of powertrain and energy balance to recover an equipment fault on a marine vessel
}

\author{
Jani Alho Tuomo Lindh Pasi Peltoniemi Jan-Henri Montonen \\ Andrey Lana Antti Pinomaa Olli Pyrhönen
}

LUT School of Energy Systems, Lappeenranta-Lahti University of Technology, FI-53851 Lappeenranta, Finland. E-mail: $\{$ jani.alho, tuomo.lindh, pasi.peltoniemi, henri.montonen, andrey.lana, antti.pinomaa,olli.pyrhonen\}@lut.fi

\begin{abstract}
This paper proposes a generally applicable method of optimizing the power and energy balance of the main vessel producer and consumer units. The method is also used after an equipment failure as part of the power management process flow. The method proposes that the route plan is divided into distance steps and an optimization algorithm is used to determine the optimal way of producing power, deploying energy storage systems and controlling propulsion. The algorithm is used to autonomously determine the estimated vessel speed. By using additional inputs to the functions it is possible to assess the weather conditions and their effect on the travel plan, and thus always provide the most optimal solution to reach the harbor.
\end{abstract}

Keywords: marine vessel, hybrid electric, fault recovery, redundancy, optimization

\section{Introduction}

As the current trends of development in all fields of mobility focus on energy efficiency and emissions reduction, also the marine industry is rapidly developing new hybrid and full electric systems to comply with the stricter regulations and to preserve our environment (Dedes et al., 2012). Networking and cloud services may vastly improve the overall efficiency of modern marine systems, and the more autonomous control devices being developed may also contribute towards the achievement of the development goals. (Vagia et al., 2016; Munin, 2018).

The benefits of hybridization are based on peak shaving and being able to more freely operate internal combustion engines in load points with more optimal fuel consumption (Sciberras and Grech, 2012). Moreover, it is possible to avoid unnecessary acceleration of the engines and run them with less emissions. This also benefits the mechanical life span of the internal combustion engine (ICE) (Geertsma et al., 2017; Lana et al., 2012).
The complexity of the hybrid system adds up to more costs and weight. Weight has adverse effects on the efficiency of the vessel payload. Ships sailing long distances may also benefit significantly from the wave load compensation implemented with rechargeable energy storage systems (RESS), which helps to maximize the engine life span and optimize the load point (Lana et al., 2019). There are also other new technologies, such as rotor sails, to boost efficiency especially in longdistance sailing (Kornei, 2017).

Technology is developing rapidly, and marine vessel scale units are becoming more available and accessible for shipyards. It is also a chance for new system providers that may bring more economical or more flexible solutions to the market. In addition to designing new systems, retrofits of vessels already in service are also being carried out (Jaurola et al., 2019). Not only hardware is required, but increasingly complex software solutions are needed to control the onboard technology in an efficient way (Lana et al., 2019; Skjetne and Sørensen, 2004). 
This sets new requirements for the robustness of automation, more specifically, the robust control of the vessel powertrain, which has been studied here as part of a project called INTENS (Integrated Energy Solutions to Smart and Green Shipping). This paper discusses the principles of a robust control method that is used to define control references for the available equipment. The travel plan must always comply with the safety requirements and environmental operational limits and simultaneously provide an efficient plan for the economical use of the vessel. The problem is simplified to a cost optimization problem.

In addition to optimizing the efficiency, there have to be methods to safely handle fault situations. The demand for such methods is acute as new vessel powertrain system topologies have become more complex with different hybrid and full electric systems, and efficient control of the system by the user or users is not always assured. With the development of autonomous vessel control systems it is even more crucial to be able to mitigate fault situations with respect to optimal efficiency (Thieme and Utne, 2017).

Optimizing the travel plan in a time-stepping manner has been considered, and it has been shown that the method is applicable to analyze and plan the use of the vessel when typical consumption data or estimates are known Ritari et al. (2019). With this approach, the location of the vessel is calculated in relation to time and vessel speed. The method suggested here, however, optimizes power production over a known route divided into distance-based segments and minimizes the operational expenditure (OPEX) of the vessel. The process values are updated and reoptimized upon the changing of external (e.g., route change) or internal (e.g., equipment failure) conditions. The fundamental idea is that the route and its constraints are static and it is possible to vary the driving parameters and thus simplify the object of optimization. Handling of faults can also be accomplished by simple logical operations, but in order to provide an optimal solution to prevent delays in service, saving fuel and minimizing operating costs are also considered crucial. Ritari et al. (2020) have investigated a time-stepping method based on predetermined vessel speed obtained from collected vessel use data. The methodology also utilizes a rule-based logic to better match unexpected changes in the power demands. By optimizing the system control strategy as distance-steps it is possible also to optimize the vessel speed as well, and similarly, a rule-based logic handles the acute power production changes locally.

The objective of this paper is to investigate optimization of the operating plan of a vessel powertrain system as a function of cost derived by power references versus distance. The actual optimization process can be cal- culated using different optimization methods; however, an analysis of the efficiency of different methods was not within the scope of this study.

\subsection{Vessel powertrain system example}

The system design of a modern vessel consists of multiple power producer and consumer units. It is possible that a shaft generator is used as a propulsion motor and vice versa, and that there are power storage units in the system. To efficiently control such a system, the use of the subsystems must be optimized. Information about equipment efficiency is crucial for creating cost functions. This, in particular, concerns the internal combustion engine and its control strategy, for which the information is obtained from brake-specific fuel consumption data and emissions analysis. For the calculations suggested here, the system can be simplified as in Figure 1, where the system consists of a DC distribution system, two diesel generators, a hotel grid, and a battery unit. Such systems have become more popular, offering some additional advantages over previous topologies (Kyunghwa et al., 2018).

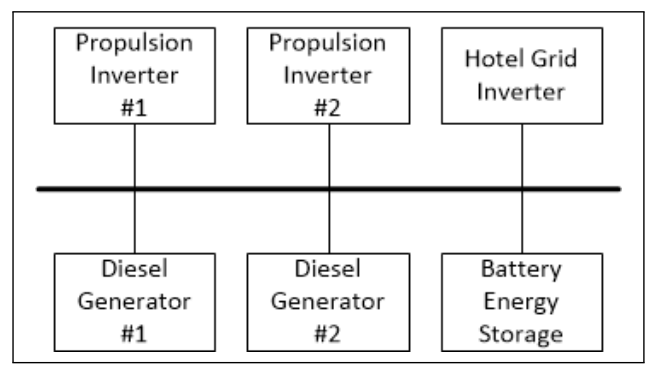

Figure 1: Illustration of possible power consumer and production units in a hybrid system based on DC distribution.

The components in the simplified system presentation represent producers (generators), consumers (hotel, other loads, propulsion), and producer-consumers (mainly energy storage). In the calculations studied here the common variable is power, and thus, the optimization problem is effectively used to solve a problem of the cost of the power balance estimation. Each component may be operating normally (online or offline), have restricted operational limits, or no availability after complete equipment failure.

\section{Overview of the optimization strategy}

The method suggests to optimize the travel plan using a cost function. Here, the cost function is a sum 
function that consists of several subfunctions, which provide the cost of use for each subsystem at a certain level of use. Several additional functions can be added to steer the optimization in the desired direction. This is explained in more detail in the following.

The travel plan is divided into distance-based steps where the optimization algorithm will decide the setpoints for the powertrain control and how to optimally control the energy balance. Depending on the complexity of the cost function and mainly the penalties or rewards formulated therein, the importance of estimating exact costs is case specific and the cost information is not open data, and not relevant to explain the proposed method. We will use weighted cost functions to direct the optimization results in a desired direction mainly to reduce unnecessary starting and stopping of engines and to direct the use of the energy storage. The route profile is known beforehand, and the optimization is carried out from the current position on the route, while reducing the optimization problem by leaving out the faulty equipment and adjusting constraints, if necessary. The optimization variables can be limited to decrease the time required to solve the problem.

In a hybrid or full electric system where a RESS is included, the optimization problem is essentially similar to the power plant problem investigated by Tiainen et al. (2008), where the main factor adding complexity is the control of the energy storage. The problems of energy storage control in a marine vessel are further discussed in Lana et al. (2012) and analyzed in more detail with generator control strategies in Lana et al. (2019). The costs of storage use must cover the lifetime costs of the RESS. When using constant-length distance steps, the equipment including an energy storage system is expected to use a constant reference for each step. Thus, the steps must be short in places where the energy storage is expected to be discharged or charged more frequently. It is to be noted that the objective of this paper is to examine the problem of control methodology rather than the dimensioning of the system components, as in Lana et al. (2019). Multiple RESS control must also take care of the balancing of the state of health figures of the energy storages. The use of RESS is becoming more important also due to requirement for partial full electric operation and other benefits obtained by using more optimal system topologies. For example, Ritari et al. (2020) has studied optimal vessel equipment dimensioning and topology suggesting optimizing ICE load points with the use of RESS and replacing some of the ICE-based reserve production capacity with RESS-based energy reserve strategy.

In this paper, a series hybrid propulsion system is observed, and the propulsion is equipped with electric machines. The starting and stopping cost of each internal combustion engine is defined, and the use costs are incorporated within the estimated lifetime costs and fuel costs.

The energy storage costs are estimated per charging and discharging power, and the final state of charge (SOC) is rewarded or penalized in the cost function. The lifetime costs of the batteries are highly dependent on the investment costs and the expected battery lifetime, in which charge cycles and use conditions play a significant role (Lana et al., 2019). This is an important matter also in the system component dimensioning, but for this problem-solving method, the storage usage function and costs must be predefined. The costs of using the electric propulsion machine were neglected in the optimization as the optimization was done from the perspective of power production versus vessel speed. Naturally, it is possible to estimate more realistic travel costs or to add other items to preserve the lifetime of other equipment.

In the simplest form, the vessel speed is estimated as a function of propeller curve, and additional forces affecting the vessel dynamics could be added as a feedback term. The produced speed is determined by the overall power allocated to the propulsion by energy balancing. This determines the speed estimate for each step of travel and accumulates the estimated travel time. The travel time has a predefined target, and arriving late will be penalized by using an additional cost term.

\subsection{Overview of the logical system design}

The route planning system must consist of the following three main stages, which can be presented as black boxes with relevant inputs and outputs:

- a system for dynamic positioning and routing, which considers the weather conditions and produces an optimal route to the destination with least resistance;

- optimization of the available resources using the route plan;

- allocating and controlling power producers and consumers to autonomously prevent a blackout in a failure situation, until an updated travel plan is available.

The interdependences of these processes are illustrated in Fig. 2. Internal decision-making process is illustrated in the next section. 


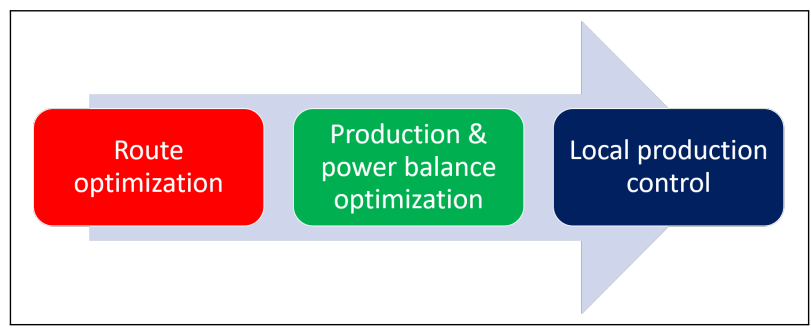

Figure 2: Optimization process flow.

In addition to automated replanning of the route, the remaining distance with the short-time average energy consumption can be offered to the crew.

\subsection{Handling of faulty equipment}

First, the optimal route from point A to point B must be determined. Then, the route optimizer occasionally reruns the optimization process if, e.g., live weather data can be taken into account when estimating an optimal route. A systematic approach could define substitute routes by determining nodes on the way from which the route to the nearest harbors will be optimized.

The predefined constant loads are prioritized so that there is an absolute reserve value for the most important consumers. For example, it is possible to design the consumer grids as a secondary level or a nonnecessity consumer level, which may have a lower priority than the propulsion. The prioritization must be implemented within the power allocating algorithm, which then takes care of the power balance equation requirements based on the current maximum power available from the power production system. The algorithm can also ensure the predefined power reserve or the minimum number of power production units online.

To prevent a blackout, a local allocation algorithm must override, if necessary, the previously optimized plan until a reoptimized operating plan matching the available equipment is available. For example, if the ship has four similar combustion engines of which two are running before a failure, and two running engines is the minimum requirement in the optimization plan, the allocation process alone will address the initial problem by starting more power production. However, the availability of each unit must be assessed and the limits used in the optimization have to be reset accordingly. As the equipment availability has changed, the optimization must be run to ensure proper operating plan for the rest of the route. In a fault case where similar equipment is available, it is likely that the plan will not be changed.

Whenever an equipment failure occurs, the power limits, or other optimization constraints, are affected. Thus, the limits for production and consumption must be specified for each case individually. For example, the storage unit power limits can be adapted or reset to zero to simulate a total failure during which the storage is offline. Whenever a fault is triggered, the availability and restrictions (or limits) of the subsystems are evaluated, and the optimization is run. The basic flowchart of a process cycle is demonstrated in Figure 3.

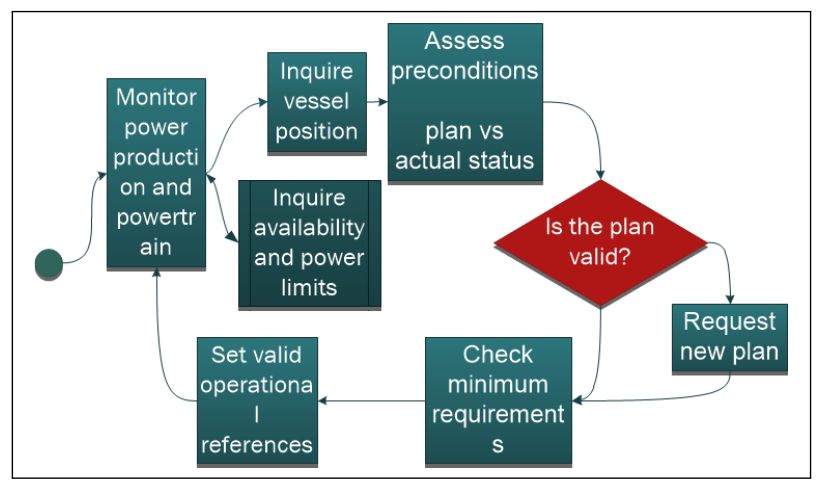

Figure 3: Logical implementation of the power allocation process plan.

The reoptimization process must determine whether the fault prevents the ship from reaching the main target harbor. In such cases, a substitute route to a harbor closer by must be selected and optimized for least travel losses. The power optimization must then determine the best way to operate on the selected route. The optimization problem will also get smaller as the vessel moves along the route, as the problem is always defined from the point of the current location on the route, using the equipment available at the moment. If there is no route optimizer available, it is also possible to give an estimate on how far the ship is able to travel with the selected speed.

\section{Optimization principle}

The travel plan is divided into segments or steps based on distance, and thus, the resolution of the algorithm depends on the length of the steps. Because an energy storage has limits regarding the capacity and charge level, the intervals must be short enough to prevent over- or undercharging, or otherwise the power references may become too low. This may happen as a result of averaging, which is caused by a low charge or discharge power, and thus limits the system unnecessarily. For example, the battery may get full during a step, and therefore, the charging must be limited. Thus, the average power for the step time is estimated. 
This, however, affects the power supply from other producers, unless the optimizer determines that the vessel speed can be kept at the same level, and thus, only the fuel consumption gets higher. In some cases, longer steps may limit the use of the power storage, so this must be taken into account when defining the route steps and setting the constraints. The same applies to ICEs, in principle, but usually the fuel storage is large enough to make this kind of observation unnecessary.

Segments with a variable distance would be useful, but it would greatly complicate the optimization. The optimization is based on estimated location per step and the time or distance covered by each step varies, producing a vast number of nonlinearly connected cases to be solved. Depending on the implementation of the algorithm, one way to address this would be to limit the number of steps, set the rules on a route look-up table, and then let the algorithm optimize the route with a free distance variable. Some decisions, however, will cause illegal overlapping of the step results. This method would allow the optimization algorithm to make a great number of illegal guesses, making the process much slower. It would require more calculating power and could be unnecessarily complicated to perform online after a component failure.

An optimization algorithm is used to determine the overall power production target so that the current static power requirement is met and the rest is allocated to electric propulsion or charging of the storage. The constraints must reflect the known or estimated future situation. The route is divided into segments covering a certain distance, and the speed limits of the route must be set. The permission to use diesel engines is also indicated for each section, e.g., in a case where full electric operation is preferred. The minimum number of power production units online can also be used as a constraint. The algorithm must determine the achievable maximum speed with the current power reserve and adjust the constraints accordingly. Optimally, the propulsion force equation would be adapted to prevailing weather conditions using an additional resistance factor.

The cost function will return the total cost, which will then be minimized. In the algorithm, several weight factors are used to either penalize or reward depending on the desired behavior. For example, energy storage levels can be appreciated in a way that the plan does not consume the stored energy fully on the route, but will try to charge so that the next trip can be started with an adequate SOC level. In this function, it is implemented as a rewarding factor, but could also be carried out by using a multivariable optimization strategy. At the beginning, the initial values of each energy storage system must be given as inputs to the first step. This applies also to a fault case, where the location of the vessel and the actual status of the components must be known to have proper initial values and correct optimization constraints.

\subsection{Object function}

The cost function is combined with the factors of the energy balance equation. Simplified functions for each device were used in the calculations.

$\begin{array}{ll}C_{\text {total }} & \begin{array}{l}\text { total costs } \\ C_{I C E}\end{array} \\ C_{R E S S} & \begin{array}{l}\text { use cost of internal combustion } \\ \text { engines } \\ \text { use costs of energy storage sys- } \\ \text { tems }\end{array} \\ C_{\text {penalties }} & \begin{array}{l}\text { total penalty costs } \\ \text { binary, engine running }\end{array} \\ V & \begin{array}{l}\text { vessel velocity } \\ \text { resistive forces affecting the ves- }\end{array} \\ R & \text { sel } \\ c & \text { ship dynamics constant } \\ p & \text { penalty factor } \\ t & \text { travel time } \\ s & \text { distance of travel } \\ a & \text { appreciation of SOC in the end }\end{array}$

Constrained variables of the object function
$P_{I C E} \quad$ power reference for the internal combustion engine (ICE)
$P_{R E S S} \quad$ power reference for the recharge- able energy storage system (RESS)

The total cost sum is calculated per steps $\in$ $\left[1 . . n_{\text {steps }}\right]$ as in the equation

$$
\begin{aligned}
C_{\text {total }} & =\left(\sum_{j=1}^{n_{\text {steps }}} C_{I C E_{j}}+C_{R E S S_{j}}\right) \\
& +\sum C_{\text {penalties }}
\end{aligned}
$$

The internal combustion engine costs include the starting cost of the engine, a cost component as a function of power used, additional running cost, and a stopping cost.

The energy storage costs represent the lifetime costs, and in this experiment they were treated as absolute values, the charging and discharging being thus equally destructive to the battery. An example of cost function: 


$$
\begin{aligned}
C_{\text {total }} & =\sum_{j=1}^{n_{\text {steps }}}\left(\sum _ { i = 1 } ^ { n _ { I C E } } \left(\text { run }_{i_{j}} \text { start }_{i_{j}} * C_{\text {start }_{i j}}\right.\right. \\
& \left.\left.+C_{P_{I C E, i_{j}}}\left(P_{i j}\right)+C_{t_{I C E}} * t_{j}\right)+ \text { stop }_{i_{j}} * C_{\text {stop }_{i j}}\right) \\
& +\sum_{i=1}^{n_{R E S S}}\left(\left|P_{R E S S, i_{j}}\right| * C_{R E S S, P_{i j}}\right. \\
& \left.\left.+a_{i} *\left(E_{\text {end }_{i}}-E_{\text {begin }_{i}}\right)\right)+\sum_{k=1}^{n} C_{\text {penalties }_{k}}\right) \\
& +C_{\text {delay }}
\end{aligned}
$$

The term $C_{\text {penalties }}$ refers to any number of additional cost factors. The operational costs of an ICE can be calculated using a figure based on running hours and then adding the fuel consumption and starting and stopping costs.

The power variables must fulfill the power balance equation

$$
P_{\text {balance }}=\sum_{i=1}^{n_{\text {units }}}\left(P_{i} * \eta_{i}\right)
$$

Another point is that relevant efficiencies should be taken into account when defining the power terms and how they relate to the equipment variables. The availability of the equipment is controlled by the power constraints. Power reserve and its logical implementation has been omitted here for simplicity. The efficiency of the power producers is also taken into account.

Generator and converter losses reduce the input power of the power balance equation versus the power reference. The efficiency of the energy storage system and its DC/DC converters must consider both charging and discharging, and the vessel speed is calculated using the effective propulsion shaft power.

\subsection{Vessel velocity and travel time}

The vessel velocity is then calculated from the power allocated to the propulsion using the propeller law equations as explained in the Basic principles of ship propulsion published by MAN. The resisting forces $R$ behave as in equation 4 , where $V$ is the ship velocity and $c$ is a ship-related constant.

$$
R=c * V^{2}
$$

With a fixed pitch propeller, the propulsion power required to achieve a certain velocity is calculated by the propeller law equation MAN Diesel \& Turbo publication (2011)

$$
P_{\text {prop }}=R * V=c * V^{3}
$$

Thus, the ship velocity derived from the propeller law equation is

$$
V=\sqrt[3]{1 / c * P_{p r o p}}
$$

and the travel time estimate follows from the vessel speed over the steps

$$
t=\sum_{i=1}^{n_{\text {steps }}} V_{i}\left(P_{\text {prop }_{i}} * s_{i}\right.
$$

The delay cost $C_{\text {delay }}$ is the sum of time consumed by each step compared with the preset target time multiplied by a penalty factor as

$$
C_{\text {delay }}=\left|M I N\left(0, t-t_{\max }\right) * p_{\text {delay }}\right|
$$

\subsection{Generator start and stop costs}

An engine start cost is added if the engine has not been running in the end of the previous step

start $_{i}=\left\{\begin{array}{l}b=0, \text { if } \text { run }_{b, i-1}>0 \text { and } 1<i \leq n_{\text {steps }} \\ b=1, \text { if } \text { run }_{b, i-1}=0\end{array}\right.$

Similarly, if the engine is not required during the step, a stop cost is added. A running condition for the step is defined as

$$
\text { run }_{i}=\left\{\begin{array}{l}
b=0, \text { if } P_{i j}=0 \\
b=1, \text { if } P_{i j}>0
\end{array}\right.
$$

\subsection{Constraint and penalty functions}

The power constraints can be defined as

$$
P_{\min _{i}} \leq P_{a c t_{i}} \leq M I N\left(P_{\text {avail }_{i}}, P_{\max _{i}}\right)
$$

Distance-based constraints to be defined are:

- Speed limits (based on geographical location or available power production);

- Use of combustion engine permitted;

- Power use: minimum and maximum power per production unit;

- Energy storage: minimum and maximum allowed charge, maximum charge, and discharge currents. 
The penalty and cost functions can be presented, where $C_{n}$ can refer to excess travel time, storage SOC out of bounds, velocity out of bounds, and additional penalties, e.g., to prevent unnecessary variance of velocity.

$$
C_{\text {penalty }}=C_{\text {delay }}+C_{n}+\ldots
$$

The number of producers online is calculated as a sum of running engines or available units (e.g., storage). This is governed in the optimization process so that each step is constrained by a certain minimum number of producers.

Target values for the optimization, which have to be adjusted because of the unavailability of certain equipment and change of boundaries, may include:

- Maximum travel time;

- Energy storage SOC in the end of the travel plan;

- Equipment costs; start cost, use cost, stopping cost.

\subsection{Decision variables}

In this experiment, the decision variables for the algorithm were the energy balance equation powers $\mathrm{P}$ for each unit. It was thus allowed to freely choose the operational logic of the generators.

$$
\begin{aligned}
& \operatorname{run}_{I C E_{i_{j}}} \quad \text { binary "run" variable to each } \\
& \text { ICE, if, e.g., engine needs to be } \\
& \text { running without load } \\
& P_{I C E_{i}} \quad \text { power to be obtained from each } \\
& \text { ICE } \\
& P_{R E S S_{i}} \quad \text { power reference of each RESS } \\
& \text { (here negative sign for discharge, } \\
& \text { positive for charge) }
\end{aligned}
$$

\subsection{Variable step length}

Variable step length was experimented, but it turned out that the optimization problem became extremely complex and slow to solve, because the boundary conditions change according to the decision variables and the behavior of the equation is not easily predictable. There are ways to simplify this by using a custom optimization function that will first rule out all the illegal cases and limit the decision variable resolution. However, on a longer journey it would require cloud computation to achieve results in an acceptable time.

\subsection{Assessment of optimization results}

Optimally, the distance-stepping drive references determined by using the cost function would be the input of a time-stepping ship model that would return more precise speed and power estimates. The speed limits and other constraints should be compared against the results provided by the model to take vessel inertia as well as roll and drag forces into account.

\subsection{Failure of the optimization process}

To prevent any failure of the optimization process, any route-related constraints affected by the limitations of energy production or propulsion power must be updated. Other rules, such as energy storage SOC appreciation, may have to be changed in order to allow more power during the emergency operation.

The optimization of such a system is a complex task, and the resulting surface is an uneven grid that may cause the optimization algorithm to stop for a local minimum. If possible, the constraint functions must be set so that the produced grid or gradient would not be extremely difficult for the optimization algorithm to be used.

The optimization process is supposed to produce the best available resolution that is also best in terms of cost. If the specification of the energy storage is too small for the intended travel plan, or in a case of fault, the energy supply must be provided by allowing the use of generators. If optimization is not successful, the constraints prevent finding an optimal solution and it may be necessary, e.g., to automatically alter the generator permissions and retry. An automatic emergency fail safe mode should thus be implemented. The speed constraints must also be adjusted if the lack of available power restricts the maximum speed under minimum level constraints. By using a carefully tuned penalty logic the algorithm will provide acceptable results.

\section{Case example}

In this case example, the route plan and ship equipment are imaginary. The case specifications cover the required parameters and setting up of the optimization problem. Electric propulsion is simplified to operate as one unit with powers summed together. To establish dual electric propulsion, both machines require individual decision variables and the energy balance must be weighted according to the value. The required propulsion power has to be optimized according to the route weather conditions. In a simplified case, each motor unit will reduce its own nominal power of the total propulsion power available.

\subsection{Power equation}

In our example calculation, we use a simple power balance equation. The parameters of each step must fulfill 
the equation. The variable $P_{\text {static }}$ represents a static load or power reserve. The intended use of the vessel and the active operating mode determine the complexity of the system controlling the power reserve.

$$
\left\{\begin{aligned}
P_{\text {balance }} & =P_{\text {static }}+P_{R E S S} \\
& -P_{\text {Prop }} \\
& +P_{\text {Genset }_{1}}+P_{\text {Genset }_{2}} \\
P_{\text {balance }} & =0
\end{aligned}\right.
$$

The use of bow and aft thrusters during maneuvering can be included in the static load estimation as a power reserve, predefined in the route plan for a specific location. The typical use is high power bursts, and the overall power consumption varies greatly according to weather conditions. The power balance is guarded inside the cost function and penalized if not zero.

\subsection{Vessel speed}

The vessel speed is obtained by using the propeller curve. In reality, a more complex model could be used to cover the compensation of lateral forces and to more realistically model the acquired speed. In our case, the propulsion costs were omitted and only the availability was observed. The load caused by waves can be estimated and averaged in the resisting forces added to the power-to-speed conversion. It is also possible to combine a time-stepping simulation of the route to include scheduled events, e.g., a restaurant causing more power consumption at certain times, but this will further complicate the optimization process.

To achieve a more structured approach, implementation of the control layers and subcontrollers is required. As pointed out before, these controllers are responsible for internally optimizing the energy production look-up tables for decision-making. Allowing subcontrollers to act according to predefined look-up-tables, optimization of the propulsion power requirement can be carried out more efficiently with fewer decision variables.

\subsection{Route constraints}

The route constraints consist of speed limits over the travel steps. The speed constraints are illustrated together with the result speed estimates in Figure 6.

\subsection{Other factors for calculation}

The collection of parameters includes

- genset power range, production costs, production efficiency;

- storage capacity, power limits, operating costs, efficiency;
- vessel dynamic value and propulsion efficiency;

- route specification as length per step, permitting of diesel operation, and minimum and maximum speed accordingly.

\section{Results}

The optimization method used for this study was a genetic algorithm. The specific requirements for the method of optimization depend on the implementation and complexity of the cost function terms.

Table 1 shows the nominal values of each power train unit and Table 2 the power constraints and trip-related values.

Table 1: Parameters

\begin{tabular}{|lrr|}
\hline Varible & Description & Value \\
\hline $\mathrm{c}$ & ship dynamic constant & 0.045 \\
$P_{R E S S_{\text {in }}}$ & nominal charge power & $800 \mathrm{~kW}$ \\
$P_{R E S S_{\text {out }}}$ & nominal discharge power & $2400 \mathrm{~kW}$ \\
\hline
\end{tabular}

Table 2: Constraints

\begin{tabular}{|c|c|c|}
\hline \multicolumn{3}{|c|}{ Power system constraints } \\
\hline$P_{\text {genset } 1}$ & & $0 . .3000 \mathrm{~kW}$ \\
\hline$P_{\text {genset } 2}$ & & $0 . .3000 \mathrm{~kW}$ \\
\hline$P_{R E S S}$ & & $-2400 . .800 \mathrm{~kW}$ \\
\hline$E_{R E S S}$ & $(\mathrm{SOC})$ & $210 . .1050 \mathrm{kWh}$ \\
\hline \multicolumn{3}{|c|}{ Trip-related boundary conditions } \\
\hline \multirow{2}{*}{$\begin{array}{l}\text { division steps } \\
\text { time target }\end{array}$} & & 20 \\
\hline & & $6 \mathrm{~h}$ \\
\hline \multirow{2}{*}{$E_{R E S S} / \mathrm{SOC}$} & at start & $1040 \mathrm{kWh}$ \\
\hline & end target & $500 \mathrm{kWh}$ \\
\hline
\end{tabular}

Fig. 4 demonstrates the original route plan and Fig. 5 the plan after losing one power producer in the middle of the trip.

It can be seen in Figure 6 that the estimated vessel velocity is not greatly affected as long as adequate power production is available. The chosen velocity is a result of rewarding arrival on time and penalizing delay. In this experiment, the method was able to provide a valid substitute travel plan with equal travel time.

As can be seen from the figures, the method provides adequate results, still being able to fulfill the route and time constraints. It was noted, however, that poor tuning of the awarding and penalizing factors or poor design of the generator cost functions may lead to 


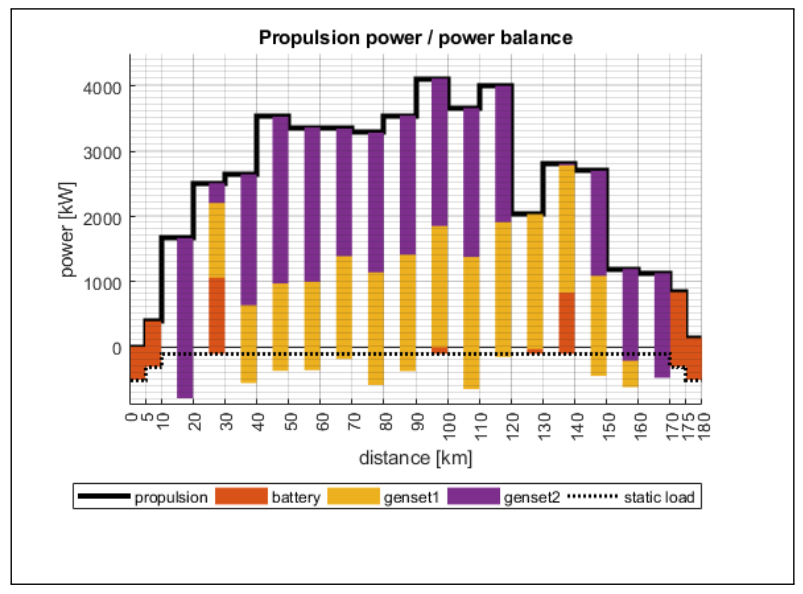

Figure 4: Original power optimization plan.

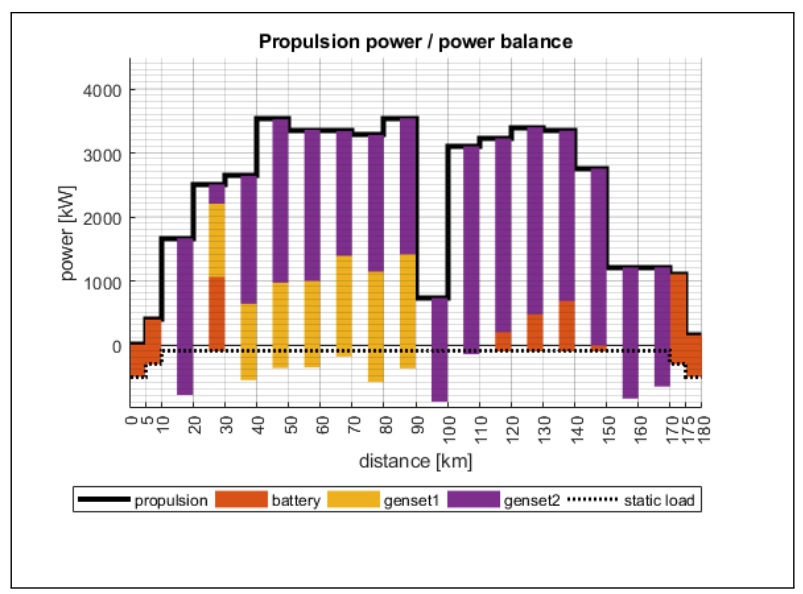

Figure 5: Example of the power optimization plan modified amid the route after losing genset 1 halfway.

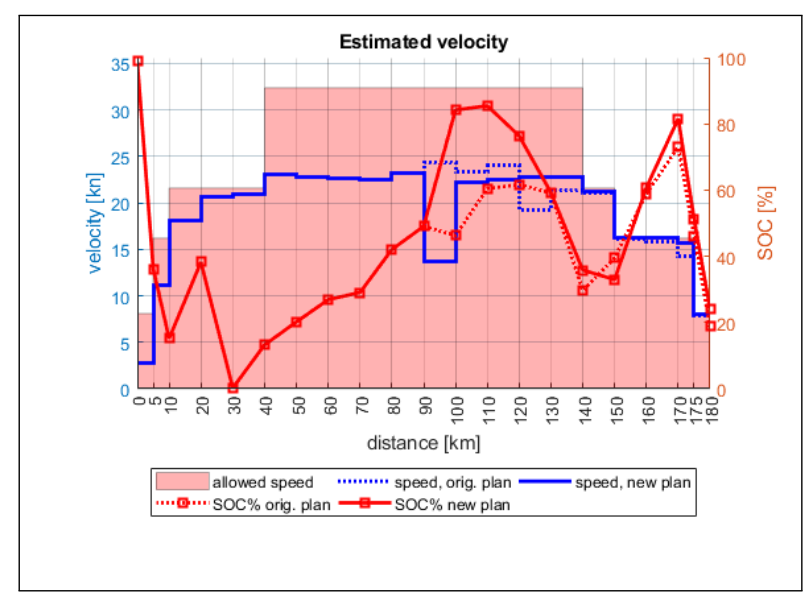

Figure 6: Estimated peak velocity and SOC\% for each segment; original travel plan and substitute plan with equipment failure halfway. seemingly irrational decisions by the algorithm. In its simplest form, the method is insensitive to changes in vessel velocity, which in some cases can lead to acceleration and deceleration during the travel. Because of the energy storage systems, the route sections may not be directly sorted to minimize the velocity changes.

\section{Discussion}

In an equipment failure, the power production and powertrain management must be capable of providing a feasible solution first to prevent a blackout and then to preserve as normal operation as possible. This is achieved by using a local powertrain and production manager, which will always protect the vessel power production according to local control position requirements (e.g., operator lever), and an onboard or remote calculation server, which will update the drive plan according to the resources available in the vessel powertrain and production.

In the case of a wider equipment failure (depending on the vessel topology), and after the local management has dealt with the acute situation, the management layer must adapt and provide a new driving scheme by which the operation can be continued in an efficient way.

Reoptimization is carried out from the current position observing the availability of the equipment, possibly taking into account additional conditions (e.g., weather). Ideally, the actual optimization system should have a database or web of route options, which is optimized by using weather data, and use routerelated information to calculate the route dynamics and then optimize the most viable control strategy for the powertrain management. The use of constraints must be considered carefully. For example, it may be sensible to not penalize the delayed arrival if there is a major loss of production affecting the maximum vessel velocity.

As the basic methodology seems plausible, the optimization method should be extended to include determination of the route distance resolution and step lengths to further enhance the optimal behavior of the energy storage system. This would require cooperation of different individual systems, and thus, significant computational resources. Naturally, it would be ideal to perform the calculation on a cloud server or the like and provide the updated optimal route plan for the vessel system so that the control system or crew can take appropriate measures.

Basically, the method proposed to optimize the power production aims at an average velocity of the vessel over a certain distance. The resulting power demand offers an average of the estimate for the trip seg- 
ments. In reality, the propulsion power fluctuates if driven with a speed reference, and thus, there must be reserve to take care of the energy required by different wave and wind conditions. On the other hand, if driven with a power reference, the speed fluctuates. Therefore, in its simplest implementation, the current estimation works on the principle of average power. Ideally, a ship model would be included that could further estimate the power requirements and vessel velocity against the limitations and driving conditions.

\section{Conclusions}

Using distance steps in optimization makes the optimization relatively fast and easy to manage as the selected route and its constraints are typically static constants, also in the case of an equipment failure. The only exception would be a case of rerouting. In a timestepping approach, each step of distance would be variable and extend the complexity of the optimization.

The actual acceleration and its effects on the costs of travel were not considered important in this study, as the distance of the steps would more or less average the behavior of the vessel. For example, for a small vessel in the case of a $5 \mathrm{~km}$ step, the required power can be estimated by using step power references.

The use of different values in the penalizing functions significantly affects the optimization results, and thus, the parameters must be carefully tuned for each system. This is especially true for the engine costs. In the case of faulty equipment, some constraints may have to be loosened or forgotten, and time limits may also have to be adjusted or at least the current top speed has to be used to calculate the minimum time frame for the route.

The approach taken in this paper is relatively simple to implement and was found feasible. It is possible to control multiple generators as a combined power plant and by dividing the solution of the optimization problem into several control layers.

\section{Citations}

\section{References}

Dedes, E. K., Hudson, D., and Turnock, S. Assessing the potential of hybrid energy technology to reduce exhaust emissions from global shipping. Energy Policy, 2012. 40(1):204-218. doi:10.1016/j.enpol.2011.09.046.

Geertsma, R., Negenborn, R., Visser, K., and Hopman, J. Design and control of hybrid power and propulsion systems for smart ships: A review of developments. Applied Energy, 2017. 194:30 - 54. doi:10.1016/j.apenergy.2017.02.060.

Jaurola, M., Hedin, A., Tikkanen, S., and Huhtala, K. Optimising design and power management in energyefficient marine vessel power systems: a literature review. Journal of Marine Engineering and Technology, 2019. (18). doi:10.1080/20464177.2018.1505584.

Kornei, K. Spinning metal sails could slash fuel consumption, emissions on cargo ships. Science, 2017. doi:doi:10.1126/science.aap8915.

Kyunghwa, K., Kido, P., Gilltae, R., and Kangwoo, C. Dc-grid system for ships: a study of benefits and technical considerations. Journal of International Maritime Safety, Environmental Affairs, and Shipping, 2018. 2(1):1-12. doi:10.1080/25725084.2018.1490239.

Lana, A., Pinomaa, A., Peltoniemi, P., Lahtinen, J., Lindh, T., Montonen, J.-H., Tikkanen, K., and Pyrhönen, O. P. Methodology of power distribution system design for hybrid short sea shipping. IEEE Transactions on Industrial Electronics, 2019. 66(12):9591-9600. doi:10.1109/TIE.2019.2892665.

Lana, A., Tikkanen, K., Lindh, T., and Partanen, J. Control of directly connected energy storage in diesel electric vessel drives. pages DS1e.7-1, 2012. doi:10.1109/EPEPEMC.2012.6397247.

MAN Diesel \& Turbo publication. Basic principles of ship propulsion. 2011 .

Munin. Maritime unmanned navigation through intelligence in networks. http://www.unmannedship.org/munin, 2018. URL http://www. unmanned-ship.org/munin. Accessed: 2018-11-12.

Ritari, A., Huotari, J., Halme, J., and Tammi, K. Feasibility analysis of a battery system for a passenger ferry. 2019. Isbn: 978-1-9095-2251-0.

Ritari, A., Huotari, J., Halme, J., and Tammi, K. Hybrid electric topology for short sea ships with high auxiliary power availability requirement. Energy (the International Journal), 2020. 190. doi:10.1016/j.energy.2019.116359.

Sciberras, E. and Grech, A. Optimization of hybrid propulsion systems. TransNav, the International Journal on Marine Navigation and Safety of Sea Transportation, 2012. 6(4):539-546. ISBN: 20836473. 
Skjetne, R. and Sørensen, A. Computer-based systems on ships and offshore vessels: The software problem ++.2004 .

Thieme, C. A. and Utne, I. B. A risk model for autonomous marine systems and operation focusing on human-autonomy collaboration. Proceedings of the Institution of Mechanical Engineers, Part O: Journal of Risk and Reliability, 2017. 231(4):446-464. doi:10.1177/1748006X17709377.

Tiainen, R., Lindh, T., Ahola, J., Niemelä, M., and Särkimäki, V. Energy price-based control strategy of a small-scale head-dependent. hydroelectric power plant. Renewable Energy and Power Quality Journal, 2008. 1. doi:10.24084/repqj06.345.
Vagia, M., Transeth, A. A., and Fjerdingen, S. A. A literature review on the levels of automation during the years. what are the different taxonomies that have been proposed? Applied Ergonomics, 2016. 53(Part A):190-202. doi:10.1016/j.apergo.2015.09.013.

\section{Acknowledgments}

This work was supported by Business Finland funded research-industry collaborative project consortium INTENS. 